REVIEW

\title{
House dust mite control measures in the treatment of asthma
}

\author{
Gillian Vallance' \\ Charles McSharry ${ }^{2}$ \\ Stuart Wood ${ }^{3}$ \\ Neil C Thomson' \\ 'Department of Respiratory \\ Medicine, and ${ }^{2}$ Department of \\ Immunology, Division of Immunology, \\ Infection and Inflammation. \\ University of Glasgow, Glasgow, UK; \\ ${ }^{3}$ Section of General Practice, Division \\ of Community-Based Sciences, \\ University of Glasgow, Glasgow, UK
}

\begin{abstract}
Sensitization to the house dust mite (Dermataphagoides pteronyssinus) (HDM) is the most common risk factor associated with the development of asthma in adults and children. The effectiveness of HDM control measures in the treatment of asthma is not yet proven. The strategies for control for avoidance depend on our understanding of the biology of the HDM. The evidence suggests a favorable effect of transferring allergic asthmatic children to naturally low dust mite environments, such as at altitude or in hospital, but little to suggest that this can be replicated in general practice by simple practical measures such as mattress covers. However, a recent multi-allergen reduction approach has suggested benefits may be achievable. HDM densities tend to be high in warm, humid conditions in the home, which may be modified by external factors, such as ventilation. However, ventilation control to reduce indoor humidity has had inconsistent effects on dust mite levels and asthma. The challenge is to further refine the interventions in large placebo-controlled trials such that clinical outcomes may be more easily demonstrated.
\end{abstract}

Keywords: house dust mite, asthma, allergy, control

\section{Introduction}

Asthma is a common chronic disease, affecting 300 million people worldwide. It is diagnosed on the basis of symptoms of wheeze, dyspnoea and cough and by evidence of variable airflow obstruction. There has been an increase in prevalence over the last 30 years, despite progress in understanding of pathogenesis and improved treatment. The reason for the increase is unknown, but is unlikely that genetic susceptibility has changed within a single generation, but is being unmasked as a result of environmental changes (Frew 2003). It is important to identify these environmental triggers as they may be amenable to change, and may reverse the rapid increase in asthma. Environmental manipulation may even have a therapeutic effect on established disease. The house dust mite (HDM) is an environmental agent commonly associated with asthma. The aim of this review is to discuss the evidence for the efficacy of HDM avoidance in the treatment of asthma.

\section{Atopy and asthma}

Rackeman classified asthma as intrinsic or extrinsic (Rackeman 1947). He noted that extrinsic asthma started before the age of 30 years. Now called allergic asthma, it is associated with atopy, the genetic predisposition for the development of an immunoglobulin E (IgE)-mediated response to common aeroallergens. IgE is the class of immunoglobulin that has protective antibody activity, usually against parasitic antigens. It may cause inappropriate inflammatory reactions when the antibody activity is directed against innocuous environmental proteins such as pollen, causing hay fever. Intrinsic or non-allergic asthma starts in middle age and is not associated with atopy. 
Atopic sensitization to the HDM (Dermataphagoides pteronyssinus) is commonly associated with asthma. Dermatophagoides pteronyssinus (Figure 1) is a pyroglyphid mite, described as the source of common house dust allergen in 1964 (Voorhorst et al 1964). The life cycle of the HDM is critically affected by ambient relative humidity and temperature (Wharton and Richards 1978).

The importance of the HDM in a West of Scotland general allergy clinic population is shown in Figure 2. Three quarters of the atopic subjects are sensitive to HDM, half to grass pollen, one quarter to cat dander and about $10 \%$ to dog dander. From these proportions, most of the individuals are sensitive to a combination of allergens but HDM is the most frequent sensitizer.

\section{The association between HDM exposure and asthma}

Bradford-Hill (1965) proposed criteria for demonstrating causality between an environmental agent and a non- infectious disease. The frequent association of HDM and asthma is, in his words, "clear-cut and beyond what we would care to attribute to the play of chance". However, it does not prove causation. The association must be assessed by his criteria: strength and consistency, dose-response relationship, and of introduction or withdrawal of allergen.

\section{Strength of association}

House dust mite sensitization is a risk factor for the development of asthma in adults and children (Nelson 2000). The strength of association has been documented in numerous studies in diverse environments across the world. (Sporik et al 1990; Wong et al 2002; Emin et al 2004). For example, Sears and colleagues (1989) conducted a longitudinal study of a New Zealand birth cohort to 13 years and showed that allergies to HDM and cat were highly significant independent risk factors associated with the development of asthma.

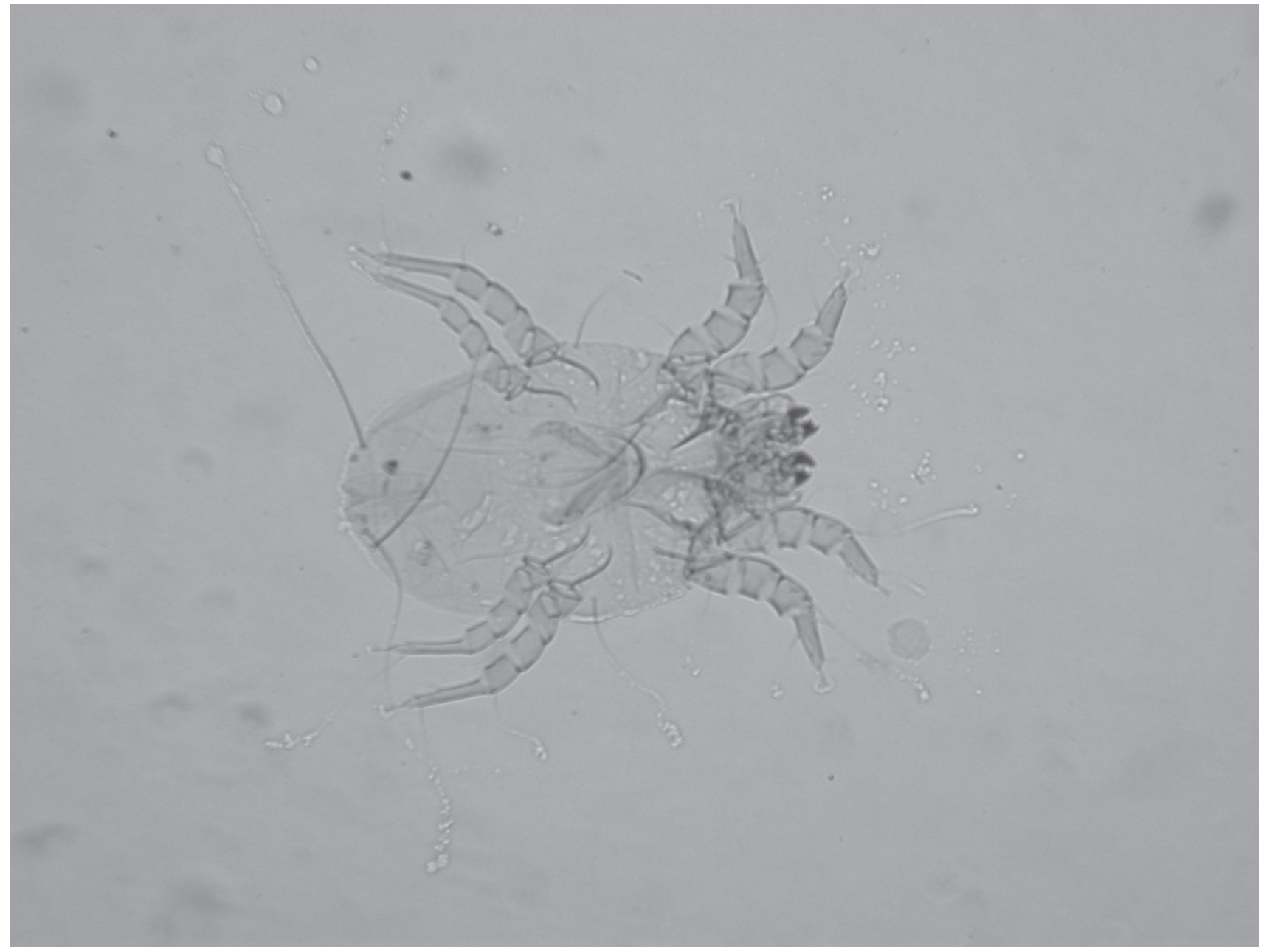

Figure I A light micrograph of a house dust mite (Dermatophagoides pteronyssinus). These mites are around $100-300 \mu \mathrm{m}$ and their secretions contain multiple soluble allergenic proteins. Glasgow University Hunterian Museum Zoology Specimen I 27238. 


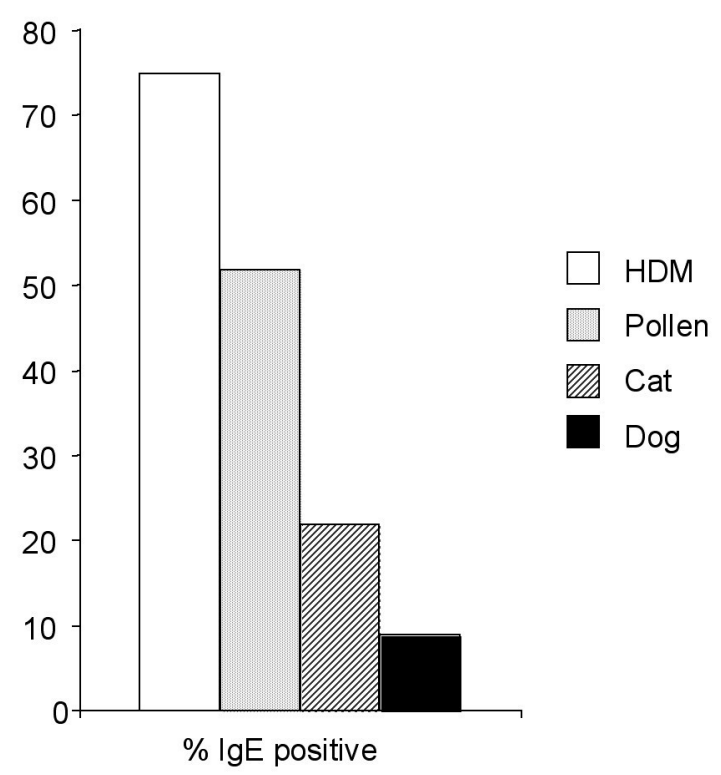

Figure 2 The proportion of atopic individuals from a West of Scotland general allergy clinic with serum IgE antibody to the allergens from house dust mite, grass, cat, and dog dander.

Abbreviations: IgE, immunoglobin $\mathrm{E}$.

\section{Dose-response relationship}

Sporik and colleagues (1990) showed that all but one of 67 children with asthma at age 11 had been exposed to the allergen Der $p 1$ at greater than $10 \mu \mathrm{g} / \mathrm{g}$ dust at the age of 1 year. The relative risk of asthma was 4.8 with the age of onset of wheeze being inversely related to the level of early exposure. In 1996, Peat and colleagues (1996) evaluated the gradation of relative air humidity and corresponding levels of domestic HDM levels in homes across 6 regions of New South Wales. As mite numbers increased there was an increased prevalence of HDM sensitization which was associated with an increased prevalence of airway hyperresponsiveness and wheeze. In 2001, the Childhood Asthma Management Program found that for HDMs, the higher the level of allergen exposure, the more likely patients were to have positive allergy skin test responses, with relative odds of 9.0 (95\% confidence interval, 5.4-15.1) for those exposed to high mite levels ( $>10.0 \mu \mathrm{g} / \mathrm{g}$ dust) relative to those unexposed. Even exposure to low levels of mite allergen $(0.020-2.0 \mu \mathrm{g} / \mathrm{g})$ was found to be a significant risk factor for sensitization (Huss et al 2001).

Custovic and colleagues (1996) found clinical activity and severity of asthma, (measured by the level of bronchial hyperreactivity [BHR], peak expiratory flow rate [PEFR] variability, and percent predicted forced expiratory volume in one second $\left.\left[\mathrm{FEV}_{1}\right]\right)$ in mite-sensitive patients, related to exposure to mite allergens in the dust reservoir.
Not all studies however find such a convincing association. Carter and colleagues (2003) found that although HDM sensitization and asthma were concurrently related, there was no relationship between level of HDM allergen exposure in children's bedrooms in early childhood and development of bronchial hyperresponsiveness or physician-diagnosed asthma by age 6 to 7 years.

\section{Allergen challenge}

Soluble extracts of house dust, HDMs or individual purified allergens from HDMs can induce an asthmatic reaction in subjects with asthma. This can be monitored as wheeze and bronchoconstriction shortly after inhaling allergen (Van der Veen et al 2001; Lophua et al 2003). This occurs in asthma patients who are already sensitive to HDM allergens, and this provides convincing evidence that exposure can cause symptoms, but does not answer the question whether exposure can induce sensitivity in a non-sensitized individual.

\section{World Health Organization recommendations}

The importance of the role of the HDM in allergic disease prompted the World Health Organization (WHO) to design guidelines for its control. It suggested that exposure to $2 \mu \mathrm{g}$ Der $p 1$ per $g$ of dust increased the risk of sensitization. Exposure to $10 \mu \mathrm{g}$ Der 1 per g of dust increases the risk of symptoms. Absolute humidity of $7 \mathrm{~g} / \mathrm{kg}$ has been stipulated as the limiting factor for the growth of colonies (Platts-Mills and de Weck 1989).

Scotland has the highest incidence of adolescent asthma in the world. We quantified the allergen content in carpets in 139 randomly selected Scottish homes. The dot plot in Figure 3 highlights the distribution of HDM allergen concentration. About two thirds of the carpets had levels above the WHO recommended sensitization limit of $2 \mu \mathrm{g} / \mathrm{g}$ (Figure 3; lower line), and about half had levels greater than the recommended clinical safety limit of $10 \mu \mathrm{g} / \mathrm{g}$ (Figure 3; higher line).

\section{Reduction of HDM exposure}

The association between HDM exposure and asthma appears to justify it as a target for control measures. However, other agents may influence lung function (Platts-Mills 2003). Cat, cockroach and other allergens, levels of endotoxin exposure and rhinovirus infection may all promote symptoms in a predisposed individual. Nevertheless, it may not be 


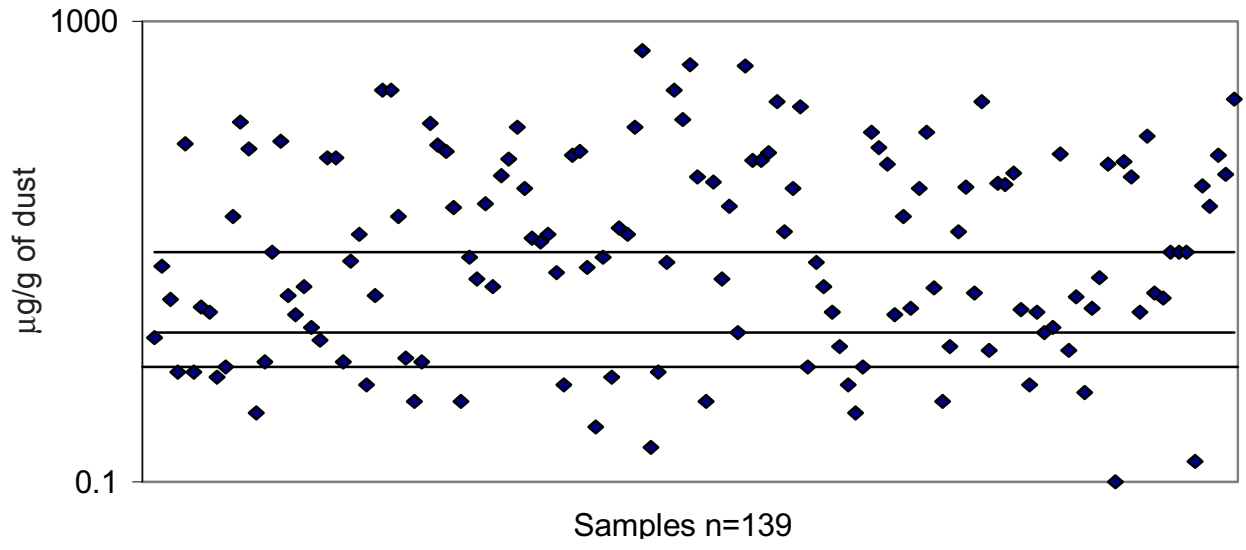

Figure 3 Dot plot illustrating the individual concentrations of the major house dust mite allergen Der pl measured as $\mu \mathrm{g}$ per $\mathrm{g}$ of settled house dust collected from carpets in 139 randomly selected houses in the West of Scotland.

necessary to prove that the HDM is the exclusive cause of asthma to demonstrate that eradication may improve symptoms. A number of observations of naturally low allergen environments have supported avoidance as an effective intervention.

\section{Altitude}

A number of studies have demonstrated the favorable effect of transferring allergic asthmatic children to high altitude Alpine sanatoria, where the natural levels of HDMs are low. Reduction in bronchial hyperresponsiveness and airway inflammation have been shown following residence at altitude (Peroni et al 1994, 2002; Piacentini et al 1999).

\section{The Tokelau hurricanes}

After hurricane damage to the Tokelau atolls in 1966, half of the population resettled in New Zealand. Children in Tokelau have a lower prevalence of asthma and atopy compared with those Tokelaun children resident in New Zealand. Lane and colleagues (2005) measured very low indoor allergen levels in Tokelau to provide an explanation for the lower prevalence of asthma and atopy, compared with New Zealand.

\section{Hospital environment}

The concept of treating patients in a mite-reduced environment has been extended to admitting asthmatic children to hospital (Platts-Mills et al 1982). The dust mite allergen levels in hospital were on average $<2 \%$ of that found at home. In this environment, clinical remission and reduction of bronchial hyper-responsiveness were noted.

\section{Application of mite control measures}

The current challenge is to replicate such low allergen environments in family homes.

House dust mite avoidance appeals as a simple, nonpharmacological maneuver, but the effectiveness of domestic eradication measures is not yet proven. Possible strategies for control will depend on our understanding of mite biology. The life cycle of the HDM is influenced by ambient relative humidity and temperature. Mites actively extract water from unsaturated air by an elegant hygroscopic pump, but water is lost due to mite activities such as feeding and defecation. The critical equilibrium is the relative humidity below which water loss is greater than water intake. Mites feed on human skin scales and are therefore abundant in bedding, carpets, soft furniture, and toys. The scales must be moist to permit digestion. Feeding rates and therefore, nutritional uptake and reproduction, are affected if the moisture content of the food is not high enough.

As relative humidity rises above the critical equilibrium humidity, feeding, mating, and egg production all increase (Arlian 1992), and as it falls, mite activity is reduced until death occurs secondary to dehydration. However, mites may be able to survive periods of dehydration if there are brief spells of elevated humidity (de Boer et al 1998).

\section{Intervention strategies to control domestic HDM numbers.}

The first example of medically instituted allergen avoidance for asthma was by Jerome Cardan in 1552. For the princely sum of 1400 gold pieces, Cardan successfully treated John Hamilton, the Archbishop of St Andrews, by advising 
removal of his feather bedding (Avenberg 1980). More recently, there have been a large number of studies examining physical and chemical methods of eradication (Table 1). The basis of these methods was varied. These ranged from using impermeable mattress covers to provide a physical barrier, chemical acaricides or liquid nitrogen, high efficiency vacuuming or improving home ventilation and humidity. Successful eradication involves both removal of residual mites and prevention of recolonization. Evidence conflicts as to whether vacuuming reduces dust mite concentration, but it is generally accepted that intensive vacuuming significantly reduces the mass of allergen in carpets (Causer et al 2004). Washing bedding at least weekly in warm water with detergent removes virtually all mite allergen. Several clinical trials of allergen-proof covers fitted to mattress and pillows have been published and the mite allergen levels in bedding could be reduced by a median of 89\% (range 39\%-99.9\%) (Eggleston 2005).

However, an objective meta-analysis of these interventions suggested that in general the evidence for their success was equivocal (Gotzche et al 1998). To make sense of seemingly inconclusive data, two measures of effectiveness must be distinguished: reduction in allergen exposure (efficacy) and impact on symptomatic illness (clinical effectiveness) (Strachan 1999).

The first Cochrane review of HDM control measures in 1998 concluded that current methods were "ineffective" and "could not be recommended" (Gotzche et al 1998). It reviewed randomized controlled trials that compared chemical or physical measures with no treatment, in patients with asthma diagnosed by a physician, and with confirmed sensitization to the HDM. Only five trials showed a reduction in mite allergen level in the intervention group. Only four of these reported changes in morning peak flow

Table I A list of physical and chemical interventions used to reduce and remove house dust mites and their allergens from the indoor environment

\begin{tabular}{l}
\hline INTERVENTION \\
\hline Physical methods \\
Barrier methods eg, impermeable mattress and pillow covers \\
Detergent and hot wash bedding \\
Steam cleaning carpets Ventilation \\
Dehumidifiers \\
lonisers \\
Chemical methods \\
Acaricide sprays eg, benzyl benzoate \\
Liquid nitrogen \\
Tannic acid \\
\hline
\end{tabular}

rate, the primary end-point. Strachan (1999) commented on these, stating that this was a conservative approach to metaanalysis, as other outcomes, such as symptomatic improvement or bronchial hyper-responsiveness, were not considered.

This was an unexpected result: firstly, as de Vet (1999) argued, it was contrary to conventional reasoning, such that reduced allergen exposure should reduce symptoms in a sensitized individual. Second, she observed that the results conflicted with a narrative review by Custovic and colleagues (1998). Of the 31 studies considered, Custovic and colleagues (1998) had eliminated those which used air ionisers or cleaners. Of the remaining 24, they excluded those which were not randomized or controlled, did not measure allergen levels, or were not measured for sufficient time. They considered 6 controlled studies to have used an efficacious intervention. There was some evidence of clinical benefit in each, such as improved lung function, symptoms, or medication use.

Thereafter, there was a call for larger and more rigorous randomized trials, particularly to evaluate methods other than those used to date. Woodcock and colleagues (2003) responded with a large randomized placebo-controlled double-blind trial to establish the effect of allergenimpermeable mattress covers on adult asthma in general practice. The key finding was that there was no significant reduction in mite allergen level at 12 months. There was no parallel change in asthma control (Figure 4). This study concluded that mattress covers, as a single intervention, seem clinically ineffective in a population of adults with asthma. The Cochrane Review included this trial in its update in 2004, which evaluated 49 trials, 31 physical methods, 10 chemical methods, and 8 using a combination of both (Gotzche et al 2004). They concluded that these methods could not be recommended for asthma. The most plausible explanation they suggested that the methods reviewed did not adequately reduce the mite allergen levels.

Morgan and colleagues (2004) designed and implemented an individualized, comprehensive environmental intervention to decrease exposure to multiple allergens including HDM in inner city children with atopic asthma. They demonstrated a significant reduction in asthma, and asthma-associated morbidity. This was a highly powered study using 937 patients in 7 inner city locations, and provided proof-of-concept evidence that environmental control of HDM could improve asthma. It would be interesting to address in future studies the issues of whether those of different severities are affected differently and the 


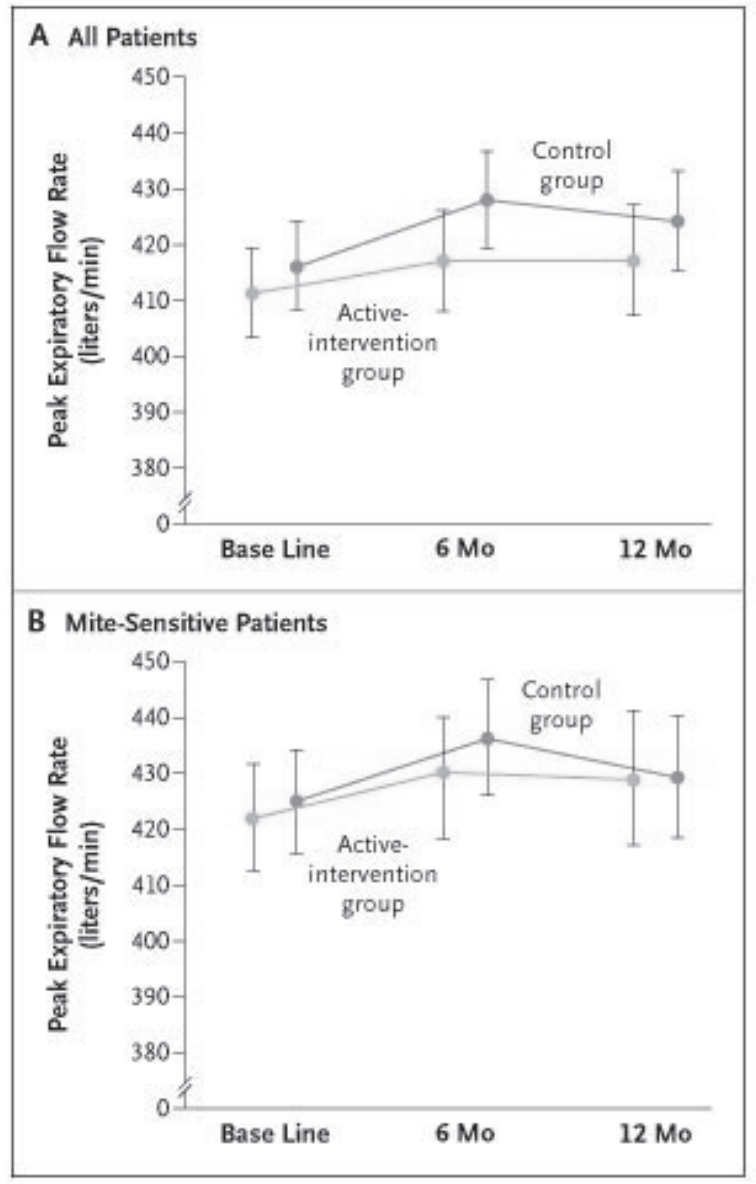

Figure 4 Mean morning peak expiratory flow rate in the Active-Intervention and Control Groups at Base Line, 6 Months, and 12 Months among All Patients (Panel A) and among Mite-Sensitive Patients (Panel B). Data points represent the geometric means, and I bars the 95 percent confidence intervals. Copyright (C) 2003. Reproduced with permission from Woodcock A, Forster, L, Matthews, et al. 2003. Control of exposure to mite allergen and allergen-impermeable bed covers for adults with asthma. N Eng J Med, 349:225-36.

complexity of the different environments of home, work, and leisure affecting any one individual.

\section{Using ventilation to control HDM}

Mite densities tend to be high in warm, humid climates. However conditions in the home may be affected by other factors such as ventilation and season. Achieving the required hygrothermal conditions to control mite populations in temperate climates may be more complex than in cool, dry climates

Ventilation control to reduce indoor humidity (dehumidifiers) has had inconsistent results. In one study, portable dehumidifiers were unable to reduce either humidity or mite allergen levels (Custovic et al 1994). In another study, mite allergen levels were unaffected by heat exchangers despite maintaining indoor relative humidity at less than 40\% (Niven et al 1999). However, Arlian and colleagues (2001) was able to maintain relative humidity at less than $51 \%$ throughout a 2 -year period in approximately half of the homes tested, and mites and mite allergen in the carpets from these homes decreased by $78 \%$.

Mechanical heat recovery ventilation (MHRV) is a method of active ventilation using both an extraction and a supply fan. The airflows are separate, but pass across a heat exchanger allowing conservation of heat energy. This was first used successfully by a Danish group led by Harving (Harving et al 1994a). Their paper suggested that HDM exposure was reduced in specially designed, mechanically ventilated 'healthy homes'. Exposure to HDMs was investigated in 30 asthmatic patients before and after moving to homes with mechanical ventilation systems. Air exchange rates increased with an associated reduction in indoor absolute humidity. There was a reduction from 110 mites/g dust to 20 mites/g dust over a 5 month period. The authors concluded that the reduction of air humidity through an increased supply of fresh air might significantly diminish and may in some cases eliminate mites in homes.

A second paper from this group (Harving et al 1994b) examined clinical efficacy in such homes, evaluating the effect on asthma control for 14 patients. After 5 months, the active subjects had improved lung function measured by $\mathrm{FEV}_{1}$, average peak expiratory flow, medicine-use score, symptom score, and serum IgE level. There were nonsignificant trends in response to histamine provocation and blood eosinophil count. A significant relation was found between reduction in medicine score and fall in HDM exposure. They concluded that, not only was MHRV effective in reducing allergen exposure, but that it had a clinically demonstrable effect on improving asthma symptoms. However, the methodology of this trial, although complex and innovative, could be criticized because there was no randomization, no blinding, and no placebo control of home-move to a non-MHRV house.

Singh and colleagues (2002) undertook a systematic review of clinical trials for humidity control for chronic asthma. This review was a search of the clinical trial registers of the Cochrane collaboration and the Cochrane Airways Group using search terms for asthma and humidity or water vapor. All randomized controlled trials on the use of humidity control measures in the home environment of patients with asthma were evaluated. The authors concluded that only one trial could be included. This was by Warner and colleagues (2000) who studied 40 patients in a parallelrandomized controlled trial over 12 months. She compared 
MHRV with high efficiency vacuum cleaning, MHRV alone, high efficiency vacuum cleaning alone, and no intervention in 4 groups of 10 patients. There was no blinding, as no placebo humidifier was used. The trial authors reported no significant differences in any of the subjective patient outcomes; however they indicated a trend towards an improvement in the response to histamine challenge. Allergen levels and HDM counts decreased in bedroom carpets, but not in mattresses and sofas. They concluded that there is requirement for a double blind randomized controlled trial with adequate sample size measuring clinical outcomes in patients with asthma.

\section{Conclusions and future directions}

The role of the indoor environment in asthma is of major concern as we spend much of our lives indoors. Asthma has become more prevalent and severe, and the majority of allergic asthmatic children are sensitive to indoor allergens.

There have been many attempts to reduce the impact of asthma by reducing indoor allergens, primarily HDM. These are difficult and costly studies and in general their efficacy has been unconvincing. Various meta-analysis and review studies have signaled that simple interventions do little to reduce HDM allergen levels. The Cochrane Review of 2004 (Gotzche et al 2004) concluded that individual chemical and physical methods cannot be recommended and suggest that studies need to use other innovative, more rigorous methodology, with clinical outcomes, and improved methods for quantifying dust mite exposure. In addition, these reviews suggest that the length of time needed for an intervention to follow through should be at least 12 months. Environmental controls are not easy, but Morgan and colleagues (2004) shows that although it is possible, further fully powered studies are required. The challenge is to further refine the interventions so that clinical outcomes may be more easily demonstrated.

This challenge is now 2-fold. Firstly to design an intervention strategy that uses the best evidence-base from the most promising published methods, and secondly to use this to impact on building regulations to encourage change in the design, construction and use patterns of domestic buildings in the UK. Since the changes to the Building Regulations in response to the energy crisis of 1974, there has been increasing resistivity of the building fabric and 'tighter' construction techniques for example, doubleglazing, central heating and changing lifestyles. These have resulted in a warmer and more humid domestic environment that is the perfect microclimate for the proliferation of
HDMs. A major international objective should be to understand and reverse this as a practical intervention in asthma.

\section{Acknowledgements}

Chief Scientist Office and Primary Care Division, Research \& Development Directorate, NHS Greater Glasgow.

\section{References}

Arlian L. 1992. Water balance and humidity requirements of house dust mites. Exp Appl Acar, 16:15-35.

Arlian LG, Neal JS, Morgan MS, et al. 2001. Lowering humidity in homes is a practical way to control house dust mites and their allergens in homes in temperate climates. J Allergy Clin Immunol, 107:99-104.

Avenberg, KM. 1980. Footnotes on allergy. 1980. Uppsala, Sweden: Pharmacia.

Bradford-Hill A. 1965. Environment and disease: association and causation? Proc Roy Soc Med, 9:295-300.

Carter PM, Peterson EL, Ownby DR, et al. 2003. Relationship of housedust mite allergen exposure in children's bedrooms in infancy to bronchial hyper-responsiveness and asthma diagnosis by age 6 to 7 . Ann Allergy Asthma Immunol, 90:41-4.

Causer SM, Lewis RD, Batek JM, et al. 2004. Influence of wear, pile height and cleaning method on removal of mite allergen from carpet. J Occ Env Hygiene, 1:237-42.

Custovic A, Simpson A, Chapman MD, et al. 1998. Allergen avoidance in the treatment of asthma and atopic disorders. Thorax, 53:63-72.

Custovic A, Taggart SC, Francis HC, et al. 1996. Exposure to house dust mite allergens and the clinical activity of asthma. J Allergy Clin Immunol, 98:64-72.

Custovic A, Taggart SC, Kennaugh JH, et al. 1994. Portable dehumidifiers in the control of house dust mites and mite allergens. Clin Exp Allergy, 25:312-16

de Boer R, Kuller K, Kahl O. 1998. Water balance of Dermatophagoides pteronyssinus maintained at brief daily spells of elevated air humidity. $J$ Medical Entomol, 35:905-10.

Eggleston PA. 2005. Improving indoor environments: Reducing allergen exposures. J Allergy Clin Immunol, 116:122-6.

Emin O, Nermin G, Ulker O, et al. 2004. Skin sensitisation to common allergens in Turkish wheezy children less than 3 years of age. Asian Pacific J Allergy Immunol, 22:97-110.

Frew AJ. 2003. Advances in environmental and occupational diseases. $J$ Allergy Clin Immunol, 113:1161-6.

Gotzche PC, Hammarquist C, Burr M. 1998. House dust mite control measures in the management of asthma: meta-analysis. $B M J$, 317:1105-10.

Gotzche PC, Johansen HK, Hamarquist C, et al. 2004. House dust mite control measures for asthma. Cochrane Database Syst Rev, 2:CD001187.

Harving H. Korsgaard J, Dahl R. 1994a. House dust mite exposure reduction in specially designed mechanically ventilated 'healthy' homes. Allergy, 49:713-18.

Harving H, Korsgaard J, Dahl R. 1994b. Clinical efficacy of reduction in house dust mite exposure in specially designed, mechanically ventilated 'healthy' homes. Allergy, 49:866-70.

Huss K, Adkinson NF Jr, Eggleston PA, et al. 2001. House dust mite and cockroach exposure are strong risk factors for positive allergy skin test responses in the Childhood Asthma Management Program. $J$ Allergy Clin Immunol, 107:48-54.

Lane J, Siebers R, Pene G et al. 2005. Tokelau - a unique low allergen environment at sea level. Clin Exp Allergy, 35:479-82. 
Lophua CE, Koopmas JG, Jansen HM et al. 2003. Similar levels of nitric oxide in exhaled air in non-asthmatic rhinitis and asthma after bronchial allergen challenge. Allergy, 58:300-5.

Morgan WJ, Crain EF, Gruchalla RS, et al. 2004. Results of a home-based environmental intervention among urban children with asthma. $N$ Eng J Med, 351:1068-80.

Nelson HS. 2000. The importance of allergens in the development of asthma and the persistence of symptoms. J Allergy Clin Immunol, 105:S628-32.

Niven RM, Fletcher AM, Pickering CAC, et al. 1999. Attempting to control mite allergens with mechanical ventilation and dehumidification in British homes. J Allergy Clin Immunol, 103:756-62.

Peat JK, Tovey E, Toelle BG, et al. 1996. House dust mite allergens. A major risk factor for childhood asthma in Australia. Am J Respir Crit Care Med, 153:141-6.

Peroni DG, Boner AL, Valone G, et al. 1994. Effective allergen avoidance at high altitude reduces allergen-induced bronchial hyperresponsiveness. Am J Respir Crit Care Med, 149:1442-6.

Peroni DG, Piacentini GL, Costella S, et al. 2002. Mite avoidance can reduce air trapping and airway inflammation in allergic asthmatic children. Clin Exp Allergy, 32:850-5.

Piacentini GL, Bodini A, Alessandro MD, et al. 1999. Allergen avoidance is associated with a fall in exhaled nitric oxide in asthmatic children. $J$ Allergy Clin Immunology, 104:1323-4.

Platts-Mills TAE. 2003. Allergen avoidance in the treatment of asthma and rhinitis. New Eng J Med, 349:207-8.

Platts-Mills TAE, de Weck AL. 1989. Dust mite allergens and asthma- a worldwide problem. J Allergy Clin Immunology, 22:416-27.

Platts-Mills TAE, Tovey ER, Mitchell EB, et al. 1982. Reduction of bronchial hyper-reactivity during prolonged allergen avoidance Lancet, ii:675-8.

Rackeman FM. 1947. A working classification of asthma. Am J Med, $33: 601-6$
Sears MR, Herbison GP, Holdaway MD, et al. 1989. The relative risks of sensitivity to grass pollen, house dust mite and cat dander in the development of childhood asthma. Clin Exp Allergy, 19:41924.

Singh M, Bara A, Gibson P. 2002. Humidity control for chronic asthma. Cochrane Database Syst Rev, 2:CD003563.

Sporik R, Holgate ST, Platts-Mills TA, et al. 1990. Exposure to housedust mite allergen (Der p I) and the development of asthma in childhood. A prospective study. $N$ Eng J Med, 323:502-7.

Strachan DP. 1999. House dust mite allergen in asthma: benefits unproven, but not yet excluded. BMJ, 317:1096-7.

de Vet H. 1999. Review: Measures to control house dust mites are not effective in patients with asthma who are sensitive to mites. ACP J Club, 4:80.

Voorhorst R, Spieksma-Boezeman MI, Spieksma FT. 1964. Is a mite (Dermatophagoides sp.) the producer of the house-dust allergen? Allerg Asthma (Leipz), 10:329-34.

Van Der Veen MJ, Jansen HM, Aalberse RC, et al. 2001. Der p 1 and Der p 2 induce less severe late asthmatic responses than native Dermatophagoides pteronyssinus extract after a similar early asthmatic response. Clin Exp Allergy, 31:705-14.

Warner JA, Frederick JM, Bryant TN, et al. 2000. Mechanical ventilation and high frequency vacuum cleaning: A combined strategy of mite and mite allergen reduction in the control of mite sensitive asthma. $J$ Allergy Clin Immunol, 105:75-82.

Wharton GW, Richards AG. 1978. Water vapor exchange kinetics in insects and acarines. Ann Rev Entom, 23:309-28.

Wong GWK, Ki ST, Hui DSC, et al. 2002. Individual allergens as risk factors for asthma and bronchial hyperresponsiveness in Chinese children. Eur Respir J, 19:288-93.

Woodcock A, Forster, L, Matthews, et al. 2003. Control of exposure to mite allergen and allergen-impermeable bed covers for adults with asthma. N Eng J Med, 349:225-36. 